\title{
Abnormal Capacitance Increasing at Elevated Temperature in Tantalum Capacitors with PEDOT:PSS Electrodes
}

\author{
Qifeng Pan $\mathbb{D}^{1,2}$ Qiao Liu $\mathbb{D}^{1},{ }^{1}$ Yuanjiang Yang, ${ }^{2}$ and Dongbin Tian ${ }^{2}$ \\ ${ }^{1}$ College of Big Data and Information Engineering, Guizhou University, P.O. Box 550025, Guiyang, Guizhou, China \\ ${ }^{2}$ Xinyun Electronics Components Corporation, P.O. Box 550018, Guiyang, Guizhou, China \\ Correspondence should be addressed to Qiao Liu; liuqiao1955@163.com
}

Received 7 July 2018; Revised 26 August 2018; Accepted 12 September 2018; Published 1 October 2018

Academic Editor: Gerard Ghibaudo

Copyright (C) 2018 Qifeng Pan et al. This is an open access article distributed under the Creative Commons Attribution License, which permits unrestricted use, distribution, and reproduction in any medium, provided the original work is properly cited.

\begin{abstract}
Due to the importance of capacitance temperature stability in precise analog circuit applications, capacitance instability at elevated temperature of $125^{\circ} \mathrm{C}$ was investigated in tantalum capacitors with PEDOT:PSS counter electrodes. Capacitance-voltage measurement supposed that residual ions in the PEDOT:PSS dispersion caused an accumulation of charges at the dielectric-cathode interface which contributed to an increase in the dielectric constant and resulted in the capacitance increasing at high temperature. Based on the hypothesis, water wash process was applied and capacitance dropped significantly at high temperature. This study shows that an additional water wash process is necessary to improve the capacitance temperature stability after each dispersion dip step.
\end{abstract}

\section{Introduction}

Due to much lower equivalent series resistance (ESR) and more benign failure mode than the incumbent $\mathrm{MnO} 2$ cathode technology, conductive polymer tantalum capacitors are expanding their market share in both the commercial world and military world. The first tantalum and aluminum polymer capacitors were introduced into the markets in early 1990s [1, 2]. At that time, tantalum capacitor with conductive polypyrroles cathode outperformed polyanlines and polythiophenes [3]. Because the processing of 3,4ethylenedioxythiophene (EDOT) is much simpler than other conducting polymers and it is not classified as toxic chemical like pyrrole, Poly(3,4-ethylenedioxythiophene) (PEDOT) becomes the material of first choice for solid electrolytic capacitors.

Tantalum polymer capacitors are not without faults. It was found that the dominant failure mechanism after high temperature life testing may be capacitance loss and ESR increasing rather than dielectric breakdown [4]. At elevated temperature, the thermal stability of PEDOT is a major argument to substitute the much more stable $\mathrm{MnO} 2$ in tantalum electrolytic capacitors. The first generation of conductive polymer tantalum capacitors was limited to using temperatures of $105^{\circ} \mathrm{C}$ because it was found that some percentage of the devices would suffer steadily increasing ESR at higher temperature [5].

During the last decades, researchers from tantalum capacitor manufacturers had been dedicated to improve the thermal stability of tantalum polymer capacitors. It was learned that principle mechanism of ESR increasing after high temperature lifetime operation is oxidation of the conductive polymer [5]. As the deterioration mechanism of ESR shift was well understood, new $125^{\circ} \mathrm{C}$ and $85^{\circ} \mathrm{C} / 85 \% \mathrm{RH}$ capable of tantalum capacitors were introduced by leading tantalum capacitor manufacturers [5-7].

Besides ESR increasing and capacitance loss after longterm operating at high temperature, tantalum polymer capacitors also suffer electrical parameter deviation at elevated temperature, such as capacitance instability and DC leakage current increasing. The capacitance of tantalum polymer capacitors can shift dramatically with the change of temperature and $\mathrm{dc}$ or ac bias. For example, the capacitance shift of polymer technology at $125^{\circ} \mathrm{C}$ is up to the range of $+30 \%$ to $+50 \%$, while the predecessor $\mathrm{MnO} 2$ remains within $+15 \%$. For electrical parameter instability of tantalum polymer capacitors does not cause catastrophic failure in field applications, it draws less attention from researchers. 
However, applications such as VCOs, PLLS, RF PAs, and lowlevel analog signal chains are very sensitive to noise partially contributed by capacitance shift of decoupling capacitors on the power supply rail [8]. Thus, it is important to investigate the phenomenon of capacitance shift with the change of temperature, especially at elevated temperature.

\section{PEDOT:PSS Dispersion}

Deposition methods for PEDOT cathode used in electrolytic tantalum capacitors include chemical oxidative polymerization or in situ polymerization, electrochemical oxidative polymerization, and conducting polymer dispersions.

In the in situ polymerization, a monomeric precursor of the conducting polymer is polymerized by an oxidizer. Iron salt like $\mathrm{Fe}$ (III) toluenesulfonate is commonly used as oxidizer in in situ polymerization. The monomer and oxidizer can be brought either sequentially or as premixed reactive solution. There are several major disadvantages in in situ polymerization process. First, the processing time is long because for every cycle two more dips are necessary. Moreover, residuals of the oxidizer and monomer involved in the polymerization reaction can cause surface charge at the Ta2O5/PEDOT interface, affecting the barrier and resulting in high dc leakage and low breakdown voltage [9].

In electrochemical polymerization process, a monomeric precursor of the conductive polymer is polymerized at an electrode. During the polymerization, ionic dopants from the electrolyte are incorporated into the polymer. For capacitor application, first, an auxiliary electrode layer has to be deposited on the surface of the insulating dielectric. The auxiliary electrode is contacted with an external electrode. Because of the large inner surface and the high aspect ratio of the small pores, polymer built up in the porous anode body by electrochemical polymerization is quite difficult. Another disadvantage of the electrochemical process is the more sophisticated technical setup.

In order to overcome disadvantages of electrochemical or in situ polymerization and to further simplify the manufacturing process, a nanoscale conducting polymer PEDOT:PSS dispersions for the formation of the cathode layer within the porous structure of electrolytic capacitors was developed. The new technology allows for the direct deposition of the cathode layer by simple coating steps without any polymerization. Since no chemical polymerization takes place during the deposition of the conductive polymer, there are no side products like iron salts, which have to be washed out or could deteriorate the performance in the finished product [5]. The leakage current and break-down voltage of the dielectric is not deteriorated, thus leading to the introduction of a new line of high-voltage tantalum polymer capacitors with longterm reliability [10-12].

\section{Experimental}

3.1. Fabrication of Tantalum Capacitors. Tantalum powder with specific charge per volume from $15,000 \mu \mathrm{Q} / \mathrm{g}$ to $23,000 \mu$ $\mathrm{Q} / g$ was pressed with tantalum wires into rectangular pellets, with a $5.8 \mathrm{~g} / \mathrm{cm}^{3}$ green density. The pellets were then sintered in vacuum around $1450^{\circ} \mathrm{C}$ for $30 \mathrm{~min}$. The tantalum anodes were anodized in a dilute aqueous solution of $0.01 \mathrm{~mol} \%$ phosphoric at $65^{\circ} \mathrm{C}$ and then were annealed at $400^{\circ} \mathrm{C}$ for $30 \mathrm{~min}$. The formation voltage was 124 volts for $50 \mathrm{~V} 47 \mu \mathrm{F}$ and 153 volts for $63 \mathrm{~V} 33 \mu \mathrm{F}$, respectively. After annealing, a reformation process was performed at the formation voltage for $60 \mathrm{~min}$. In this study, Clevios $\mathrm{P}$ with PEDOT:PSS ratio (w/w) of 1:2.5 was applied by dipping the $\mathrm{Ta} / \mathrm{Ta}_{2} \mathrm{O}_{5}$ pellets into a waterborne dispersion and subsequent drying in air and at room temperature and then at $130^{\circ} \mathrm{C}$. The particle size $d_{50}$ of PEDOT particles in the dispersion is less than 30 $\mathrm{nm}$. Before dipping into the dispersion, porous pellets were first vacuum-pumped. The purpose of pumping process is to evacuate air that filled in small pores in porous tantalum anodes and enable the dispersion to penetrate into the pellets more easily. In the prepolymerized process, 4-6 cycles were applied to provide a maximum coverage of the tantalum pentoxide dielectric with the PEDOT particles inside and outside the porous tantalum pellets. After the coating process of PEDOT:PSS, a graphite layer was coated on the pellets and then pasted with a thin silver layer and assembly to lead frame. The capacitors were finally encapsulated with epoxy before measurement.

3.2. Measurement. Prior to starting to test, capacitors were dried at $+125^{\circ} \mathrm{C} \pm 5^{\circ} \mathrm{C}$ for $30 \mathrm{~min}$ and then restored to room temperature. Capacitance of 13 samples was measured at $25^{\circ} \mathrm{C} \pm 3^{\circ} \mathrm{C}$ and $125^{\circ} \mathrm{C} \pm 5^{\circ} \mathrm{C}$, respectively. Capacitors were brought to thermal stability at each temperature. Capacitance measurements were performed with Agilent E4980A Precision LCR Meter. Test frequency was $120 \mathrm{~Hz} \pm 5 \mathrm{~Hz}$. The magnitude of the ac voltage was 1.0 volt root mean square (rms) and the dc bias voltage was 2.2 volts. Capacitancevoltage measurement technique was used to further investigate the abnormal increasing in capacitance. Capacitance of the samples was measured at $2.2 \mathrm{~V}, 10 \mathrm{~V}, 15 \mathrm{~V}, 20 \mathrm{~V}, 25 \mathrm{~V}$, and $30 \mathrm{~V}$ bias conditions, respectively.

\section{Results and Discussion}

In this study, abnormal capacitance increasing at elevated temperature of $125^{\circ} \mathrm{C}$ was observed for polymer tantalum capacitors without water wash process as shown in Figure 1. Compared with the initial value at $25^{\circ} \mathrm{C}$, capacitance change at $125^{\circ} \mathrm{C}$ is in the range of $71 \%$ to $77 \%$ for $63 \mathrm{~V} 33 \mu \mathrm{F}$ with $15,000 \mu \mathrm{C} / \mathrm{g}$ anodes $153 \mathrm{~V}$ formation and even as high as over $102 \%$ to $122 \%$ for $50 \mathrm{~V} 47 \mu \mathrm{F}$ with $23,000 \mu \mathrm{C} / \mathrm{g}$ anodes $124 \mathrm{~V}$ formation. The capacitance change is reversible upon the temperature range from $25^{\circ} \mathrm{C}$ to $125^{\circ} \mathrm{C}$ and there is no hysteresis observed.

In Figure 2, capacitance-voltage measurement shows that the average capacitance change of all samples for $50 \mathrm{~V} 47 \mu \mathrm{F}$ decreases dramatically with the bias voltage increasing. At low bias condition $(2.2 \mathrm{~V})$, the capacitance change at high temperature was about $110 \%$. Interestingly, as the bias voltage increased to $30 \mathrm{~V}$, capacitance shift rolled off to the level of $20 \%$. The effects of bias voltage on capacitance were also found to be similar in $63 \mathrm{~V} 33 \mu \mathrm{F}$ and other type capacitors with PEDOT:PSS electrodes. 


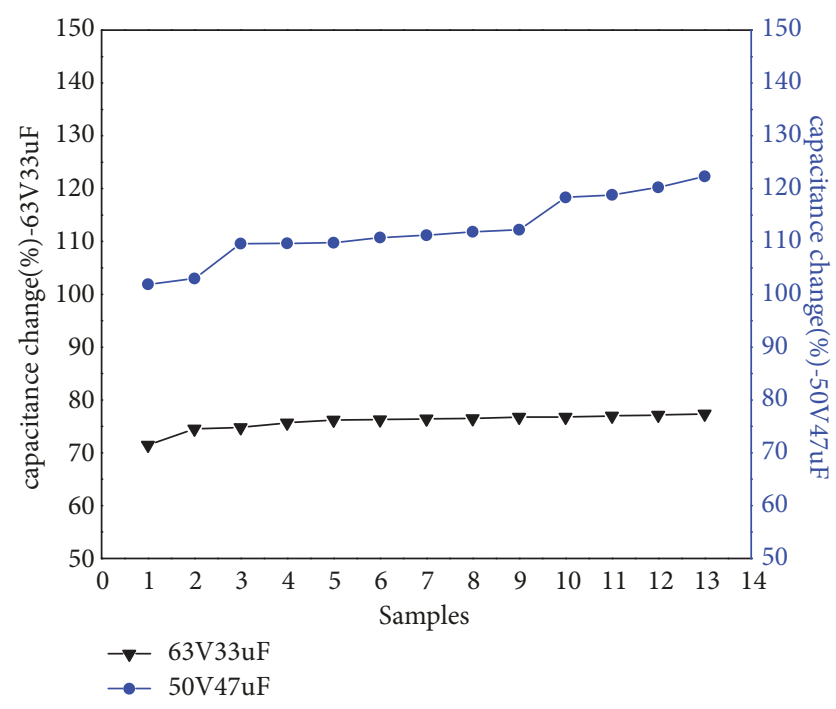

Figure 1: Capacitance change in percentage at $125^{\circ} \mathrm{C}$ without water wash process for polymer tantalum capacitors with $15,000 \mu \mathrm{C} / \mathrm{g}$ anodes $153 \mathrm{~V}$ formation for $63 \mathrm{~V} 33 \mu \mathrm{F}$ and $23,000 \mu \mathrm{C} / \mathrm{g}$ anodes $124 \mathrm{~V}$ formation for $50 \mathrm{~V} 47 \mu \mathrm{F}$.

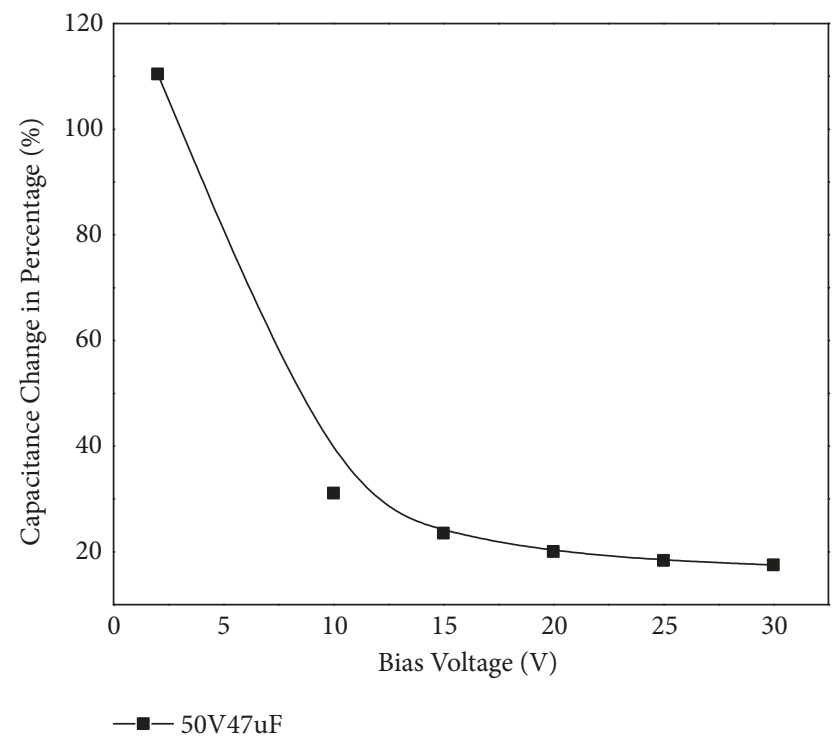

Figure 2: Capacitance-voltage characteristic of $50 \mathrm{~V} 47 \mu \mathrm{F}$ tantalum capacitors at $125^{\circ} \mathrm{C}$.

In Figure 3, the capacitance change of all samples with water wash process for $50 \mathrm{~V} 47 \mu \mathrm{F}$ decreases compared to samples without water wash steps measured at $2.2 \mathrm{~V}$ of standard bias condition. The effects of water wash process on capacitance stability were also found to be similar in $63 \mathrm{~V} 33 \mu$ $\mathrm{F}$ and other type capacitors with PEDOT:PSS counter electrode. In this study, the introduction of an additional water wash process after each dispersion dip step is to wash out residual ions with the PEDOT:PSS film.

According to capacitor theory, capacitance is determined by the following formula:

$$
\mathrm{C}=\frac{\varepsilon_{\circ} \varepsilon \cdot \mathrm{A}}{\mathrm{d}}
$$

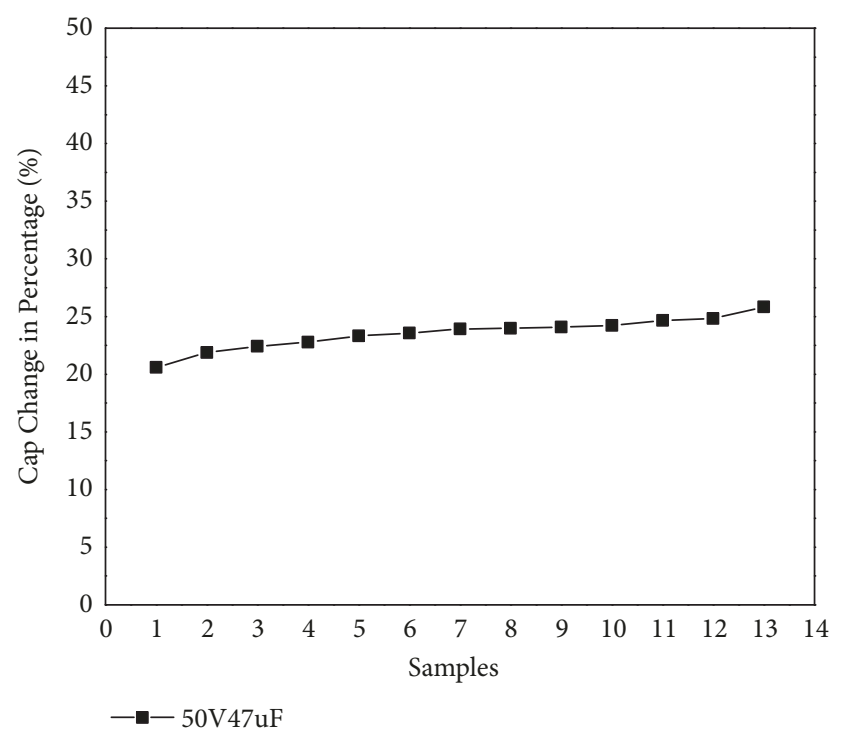

FIGURE 3: Capacitance change of $50 \mathrm{~V} 47 \mu \mathrm{F}$ at $125^{\circ} \mathrm{C}$ with water wash steps.

where $\varepsilon_{0}$ is the dielectric constant for free space $(8.855 \mathrm{x}$ $10^{-12}$ Farads / $\left.\mathrm{m}\right), \varepsilon$ is the dielectric constant for tantalum pentoxide (about 27), $\mathrm{A}$ is the surface area in $\mathrm{m}^{2}$, and $\mathrm{d}$ is the dielectric thickness in $\mathrm{m}$. In these parameters, $\varepsilon_{0}$ is a constant and does not vary with temperature and the surface area $A$ is predetermined by the tantalum powder used and the anode dimension which depends less on temperature. Furthermore, the thickness $d$ of the anodic oxide tantalum film is directly proportional to the formation voltage with the coefficient in the range of $1.6 \mathrm{~nm} / \mathrm{V}$ to $2.0 \mathrm{~nm} / \mathrm{V}$ [13]. For the coefficient of temperature expansion (CTE) of tantalum pentoxide is in the order of $10^{-5} / \mathrm{K}$, the thickness of the dielectric film will only change in the same order [14]. Thus, thickness of tantalum pentoxide is not the main cause for the increase in capacitance. Furthermore, the inherent variation of the dielectric constant for tantalum pentoxide with temperature is considerably stable within $10 \%$ at $150^{\circ} \mathrm{C}$ and would not cause abnormal capacitance increase [15]. These conclusions are verified by tantalum capacitors with manganese oxide electrode whose capacitance shift at $125^{\circ} \mathrm{C}$ is generally less than $15 \%$.

Based on experiment, Freeman, et al. introduced a model that explained the mechanism of capacitance dependency with temperature [15]. According to the model, the PEDOT layer can shrink upon cooling and expand upon heating and resulted in the changes of the effective surface area which contribute to the overall device capacitance. However, the abnormal capacitance increase observed in this study cannot be explained by the surface area model. First, the capacitance measured at $125^{\circ} \mathrm{C}$ was as high as 1.7 to 2 times compared to the value at room temperature and exceeded the wet capacitance (1.1 times the capacitance of the finished device at room temperature) measured after the dielectric formation. Practically, surface area change would not cause such a large relative change in capacitance. More importantly, strong capacitance dependency on voltage was observed at elevated 
<smiles>CC(C)[R15](=O)[O-]</smiles>

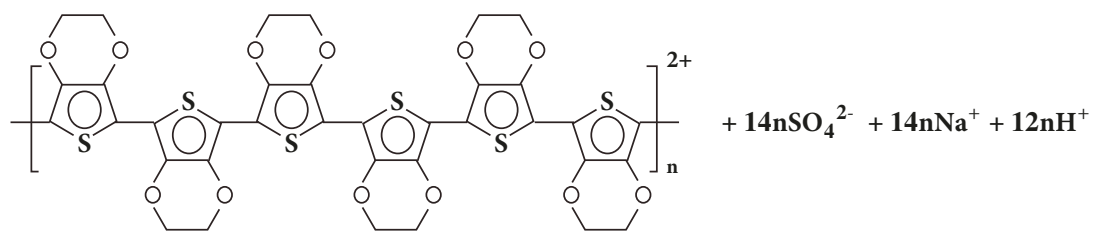

FIGURE 4: Reaction scheme for the PEDOT synthesis using Sodium peroxodisulfate as an oxidant.

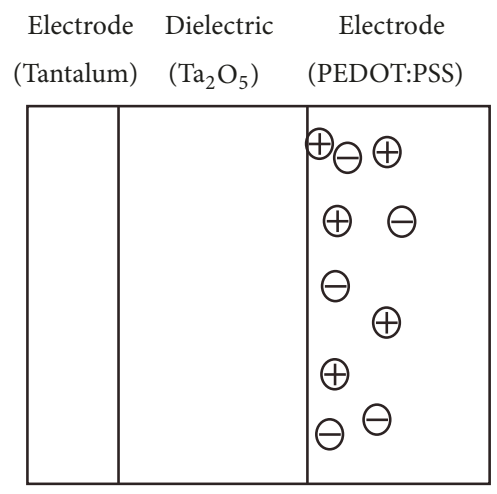

(a)

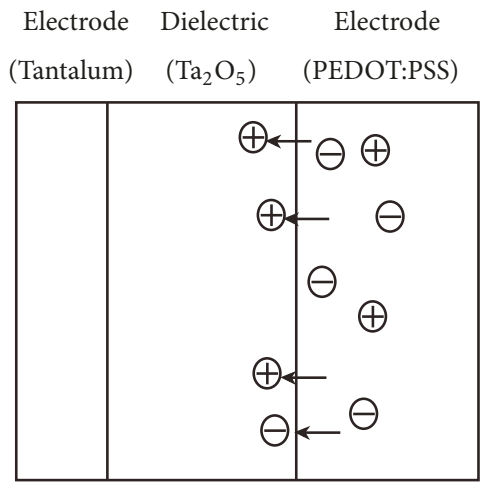

(b)



E

FIGURE 5: Interfacial polarization of tantalum capacitors with PEDOT:PSS electrode.

temperature. As seen in Figure 2, the capacitance decreased dramatically as the biased voltage increased. Obviously, the effective surface area theory cannot explain the capacitancevoltage relationship. It is believed that there are other mechanisms that may contribute to the abnormal capacitance changes.

Fundamentally, dielectric constant is a measure of dielectric polarization under an electric field. There are electronic, ionic, orientational and interfacial polarizations [16]. For tantalum pentoxide, ionic polarization is the main polarization mechanism [16]. Based on dielectric theory, both electronic and ionic polarizations are less dependent on temperature, and orientational polarization is inversely proportional to the temperature. Thus, it is supposed that the abnormal increase in capacitance may be caused by interfacial polarization due to the introduction of conductive polymer PEDOT:PSS.

The PEDOT:PSS complex was synthesized by mixing the polystyrene sulfonic acid with PEDOT. For Clevios P dispersion, the molar ratio of thiophene groups to sulfonic acid groups is 1:1.19, which corresponds to a weight ratio of 1:2.5. During the oxidative polymerization of EDT monomer in the presence of PSS, sodium peroxodisulfate is used in combination with an Fe(III) salt as a catalyst. As shown in Figure 4, the reaction mixture turns more acidic as the reaction progresses, since each mol of EDOT releases two moles of protons [3]. Theoretically, there are residuals such as protons, sodium, sulfate, $\mathrm{Fe}(\mathrm{III})$, and other species in the PEDOT:PSS complex after the completion of the synthesis.

At elevated temperature, impurities such as $\mathrm{H}+, \mathrm{Na}+$, and $\mathrm{Fe} 3+$ ions diffuse toward the interface between PEDOT:PSS cathode and tantalum pentoxide dielectrics to form an accumulation of charges; thus interfacial polarization occurs as depicted in Figure 5. Dielectric materials, although perfect, contain crystal defects, holes, and surface imperfections. As depicted in Figure 5(a), residuals in PEDOT:PSS have an equal number of positive ions and negative ions, but the positive ions are assumed to be far more mobile because they are relatively small. In the absence of electric field or at lower bias condition, positive ions may diffuse into the dielectrics of tantalum pentoxide under high temperature as shown in Figure 5(b). These positive charges accumulate at the interface and attract more electrons to the negative electrode. These additional charges on the negative electrode, of course, appear as an increase in the dielectric constant. Figure 5(c) shows that, at higher bias condition, the diffused positive ions on the dielectric side may drift back to the cathode or combination with electrons and the effect of interfacial polarization is reduced; thus, the capacitance dropped under higher bias condition as shown in Figure 2. 
TABLE 1: Characteristics of Clevios P dispersion.

\begin{tabular}{lccccc}
\hline Characteristics & min. & max. & Measured value & \\
\hline Solid Content & 1.2 & 1.4 & 1.32 & Unit \\
pH & 1.5 & 2.5 & 1.9 & \\
Sodium & & 500 & 233 & ppm \\
Sulfate & & 80 & ND & ppm \\
Iron & $/$ & $/$ & 0.64 & $\mathrm{ppm}$ \\
\hline
\end{tabular}

In order to verify the main sources that contributed to the capacitance variation, further analysis was done. For Clevios $\mathrm{P}$ dispersion used in this study, the main physical and chemical characteristics are shown in Table 1. Although $\mathrm{Fe}$ (III) salt was used as catalyst, it can be easily washed out and the residual of Fe ions is almost negligible and not specified in the specification. The main residual species given are sodium and sulfate, and the maximum contamination levels are 500 ppm and $80 \mathrm{ppm}$, respectively. In order to further analyze residual species, contents of sodium and iron were measured through leaching analysis. The contents of sodium and iron were $233 \mathrm{ppm}$ and $0.64 \mathrm{ppm}$, respectively, as shown in Table 1. As seen from the characteristics table, the specified value of $\mathrm{pH}$ for the dispersion is in the range of 1.5 to 2.5 , and the measured value is 1.9 , which indicated that the hydrogen ion concentration is high.

From the analysis and measurement, residuals in the PEDOT:PSS complex contributing to the abnormal capacitance increase at high temperature are mainly $\mathrm{H}+$ and $\mathrm{Na}+$ ions in tantalum capacitors with PEDOT:PSS electrodes.

Based on the above hypothesis, it can be assumed that an additional water wash process can reduce the content of impurity ions which resulted in the improvement of capacitance temperature stability. As shown in Figure 3, the capacitance change at high temperature decreased dramatically compared to samples without washing steps during PEDOT:PSS dispersion dipping process.

However, though an additional water wash process can improve the capacitance stability, capacitance changes at high temperature in conductive tantalum capacitors with PEDOT:PSS electrode are still higher than the conventional ones with manganese dioxide electrodes. The reasons for this larger capacitance change have already been well explained by the surface area model developed by Freeman et al. [15]. For mechanisms of capacitance dependency with temperature in PEDOT cathode system are complicated, the pore structure of the anode, preparation methods of the dielectrics of Ta2O5, the material of PEDOT used, deposition methods of PEDOT, and so forth can contribute to the capacitance variation. For the new generation tantalum capacitor technology, more efforts may be needed to further improve the capacitancetemperature stability.

\section{Conclusions}

The abnormal capacitance increase in tantalum capacitors with PEDOT:PSS electrodes at elevated temperature of $125^{\circ} \mathrm{C}$ was investigated by capacitance-voltage measurement. It was supposed that residuals such as hydrogen and sodium ions in the PEDOT:PSS dispersion caused an accumulation of charges at the dielectric-cathode interface, which appeared as an increase in the dielectric constant and resulted in the capacitance increase at high temperature. This study shows that an additional water wash process is necessary to improve the capacitance temperature stability after each dispersion dip step.

\section{Data Availability}

The data used to support the findings of this study are included within the article and can be accessed without any restrictions.

\section{Conflicts of Interest}

The authors declare that there are no conflicts of interest regarding the publication of this paper.

\section{Acknowledgments}

The authors would like to acknowledge the support of Xinyun Electronics Components Corporation for providing capacitor samples, testing facilities, and financial funding.

\section{References}

[1] Y. Kudoh, S. Tsuchiya, T. Kojima, M. Fukuyama, and S. Yoshimura, "An aluminum solid electrolytic capacitor with an electroconducting-polymer electrolyte," Synthetic Metals, vol. 41, no. 3, pp. 1133-1136, 1991.

[2] M. Fukuyama, Y. Kudoh, N. Nanai, and S. Yoshimura, "Materials Science of Conducting Polymers: An Approach to Solid Electrolytic Capacitors with a Highly-Stable Polypyrrole Thin Film," Molecular Crystals and Liquid Crystals Science and Technology. Section A. Molecular Crystals and Liquid Crystals, vol. 224, no. 1, pp. 61-67, 1993.

[3] A. Elshchner, S. Kirchmeyer, W. Lovenich, U. Merker, and K. Reuter, PEDOT: Principles and Applications of an Intrinsically Conductive Polymer. An introduction to theory and applications of quantum mechanics, Wiley, 1982.

[4] Reed, Characterization of tantalum polymer capacitors, NASA Electronic parts and packaging program, NEPP Tast 1.21.5, Phase 2,2006.

[5] E. Reed, J. Chen, J. Marshall, J. Paulsen, and R. Weisenborn, "New 125.C capable tantalum polymer capacitors," in Proceedings of the 17th Passive Components Symposium CARTS Europe, pp. 24-31, 2003. 
[6] J. Young, "Polymer Tantalum Capacitors for Automotive Applications," in Proceedings of the CARTS International, pp. 297-311.

[7] J. Petrzilek, M. Biler, and T. Zednicek, "Hermetically sealed conductive polymer tantalum capacitors," in Proceedings of the CARTS International 2014, USA, April 2014.

[8] G. Morita, "Capacitor Selection Guidelines for Analog Devices, Inc., LDOs," Tech. Rep., http://www.analog.com/media/en/ technical-documentation/application-notes/AN-1099.pdf.

[9] Y. Freeman, W. R. Harrell, I. Luzinov, B. Holman, and P. Lessner, "Electrical characterization of tantalum capacitors with poly(3,4-ethylenedioxythiophene) counter electrodes," Journal of The Electrochemical Society, vol. 156, no. 6, pp. G65-G70, 2009.

[10] U. Merker, W. Lovenich, and K. Wussow, "Conducting polymer dispersions for high-capacitance tantalum capacitors," in Proceedings of the 20th Passive Components Symposium CARTS Europe, pp. 21-26, Bad Homburg, Electronic Components, Assemblies \& Materials Association, 2006.

[11] U. Merker, W. Lovenich, and K. Wussow, "Tuning conducting polymer dispersions for high-CV tantalum capacitors," in Proceedings of the in. Proceedings of the 21st Passive Components Symposium CARTS Europe, Barcelona, 2007.

[12] J. Young, J. Qiu, and R. Hahn, "High voltage tantalum polymer capacitors," in Proceedings of the 28th Symposium for passive electronics, CARTS-USA 2008, pp. 185-195, USA, March 2008.

[13] J. P. Manceau, S. Bruyere, S. Jeannot, and A. Sylvestre, "Current instability, permittivity variation with frequency, and their relationship in ta 2 o 5, capacitor," IEEE Transactions on Device Materials Reliability, vol. 7, no. 2, pp. 315-323, 2007.

[14] Y. Freeman, P. Lessner, and E. Jones, "Low de-rating reliable and efficient $\mathrm{Ta} / \mathrm{MnO} 2$ capacitors," in Proceedings of the CARTS International 2012, pp. 19-28, USA, March 2012.

[15] Y. Freeman, I. Luzinov, R. Burtovyy et al., "Capacitance stability in polymer tantalum capacitors with PEDOT counter electrodes," ECS Journal of Solid State Science and Technology, vol. 6, no. 7, pp. N104-N110, 2017.

[16] M. Henini, Principles of Electronic Materials and Devices, S. O Kasap, Ed., McGraw-Hill, 2nd edition, 2002. 


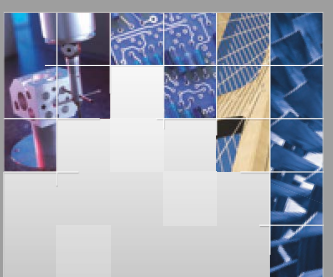

\section{Enfincering}
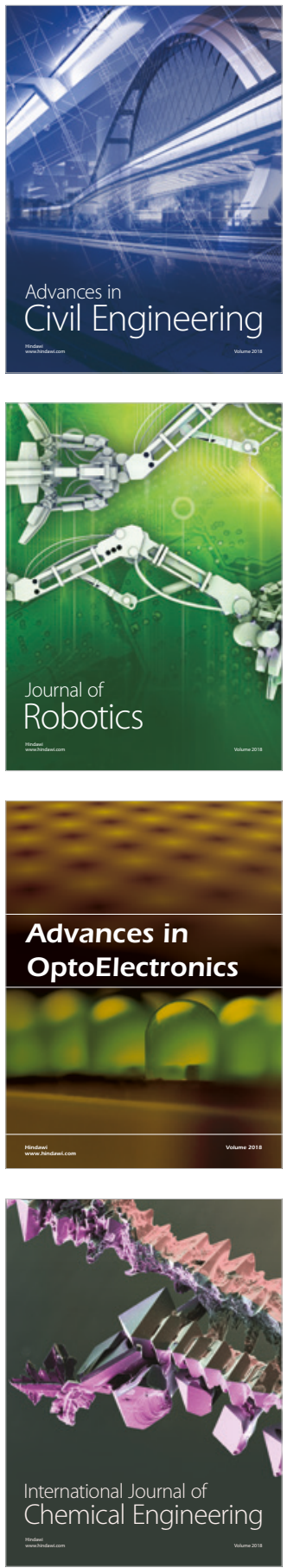

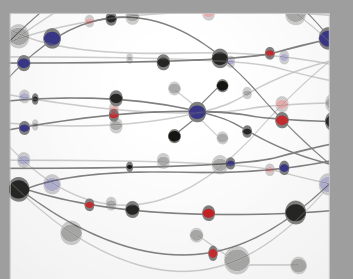

\section{Rotating \\ Machinery}

The Scientific World Journal



Submit your manuscripts at

www.hindawi.com

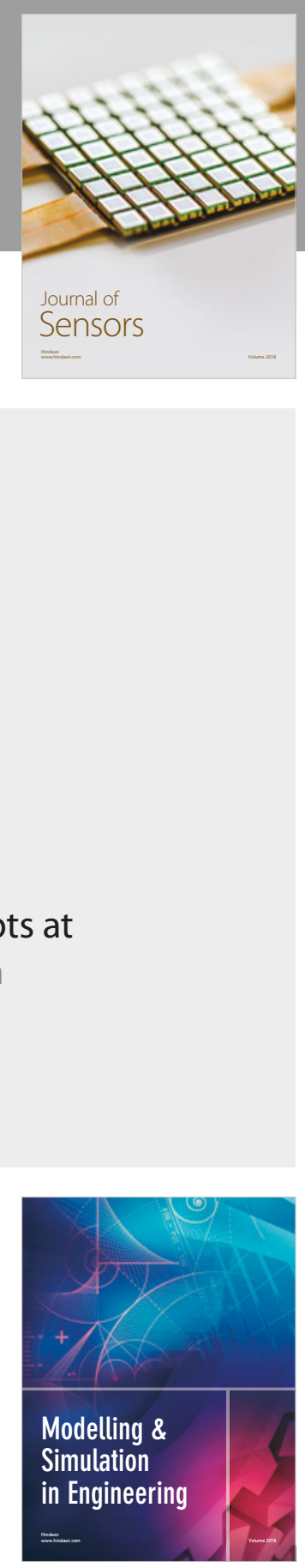

\section{Advances \\ Multimedia}
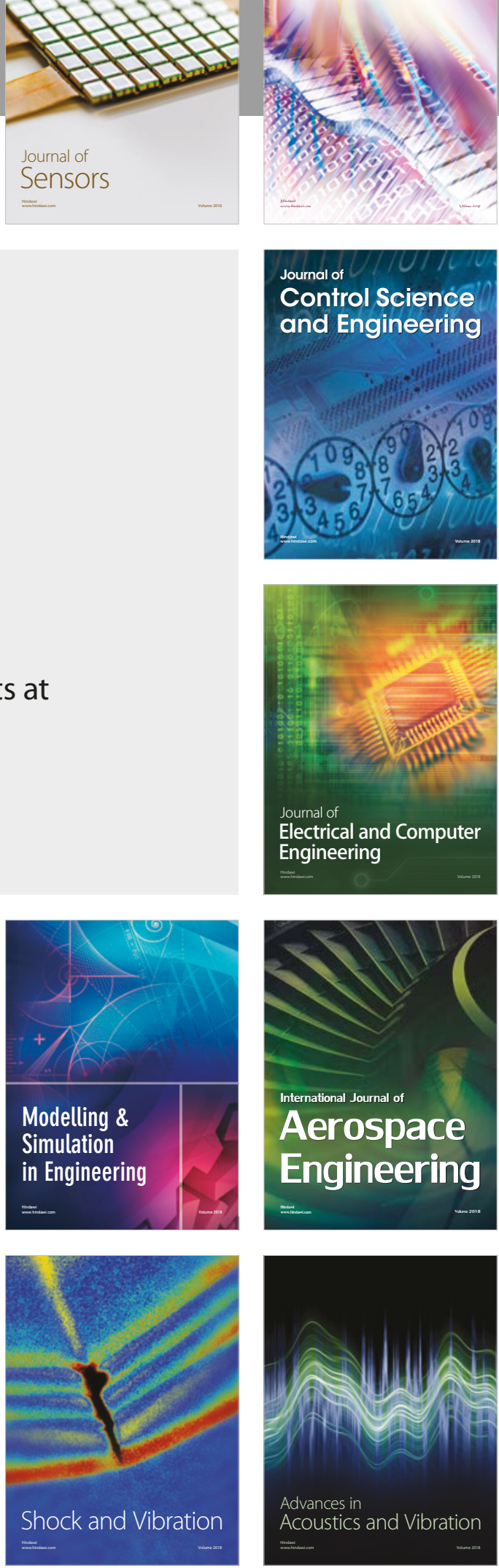